\title{
Reward magnitude and number of training trials as joint factors in extinction'
}

\author{
WINFRED F. HILL 2 , NORTHWESTERN UNIVERSITY \\ WILLIAM P. WALLACE, UNIVERSITY OF NEVADA
}

Reward magnitudes of $1,2,4$ and 8 pellets $(.045 \mathrm{gm})$ were combined factorially with $8,16,32,64$, and 128 reinforced training trials for a total of 300 rats in a straight runway. Extinction speed was a monotonic increasing function of number of training trials and also tended to increase with reward magnitude, but the latter effect was not significant. There was no interaction between reward magnitude and number of trials and no indication of nonmonotonicity for either variable.

Although the effects of reward magnitude and of number of training trials on reinforced instrumental behavior are reasonably well established, their effects on resistance to extinction remain controversial. Even when the discussion is restricted to the runway behavior of hungry rats on continuous reinforcement with solid food, conflicting results seem to be the rule. Some studies have found faster running in extinction following large reward than following small (e.g., Hill \& Spear, 1962), whereas others have found the reverse (e.g., Hulse, 1958). There are similar opposite findings regarding the effect of number of training trials (Sperling, 1965).

As Ison (1962) and others have suggested, it may be possible to reconcile the conflicting findings by considering the two independent variables of reward magnitude and number of training trials jointly. A comparison of the studies cited above suggests that reward magnitude and number of training trials are each positively related to extinction speed when the other of the two variables is at a low value, but negatively related to extinction speed when the other variable is at a high value. A paper by Ison \& Cook (1964) and pilot data from our laboratory have given some support to this hypothesis, which is further explored in the present study.

Subjects and Design.

The Ss were female albino rats of the SpragueDawley strain, 75 days old at the beginning of prehandling. There were 20 groups, representing a factorial combination of four reward magnitudes $(1,2,4$, and 8 pellets, each weighing $.045 \mathrm{gm}$ ) with five levels of acquisition training $(8,16,32,64$, and 128 rewarded trials). The Ss were run in 16 squads of 20 each, one $\mathrm{S}$ from each group.

In order to have a meaningful analysis of extinction, it seemed desirable to eliminate the data from Ss that had clearly failed to learn. In order to do so with a minimum of bias the $22 \mathrm{Ss}$ that had three nonruns on trials 5 through 8 of acquisition were excluded from the analysis. In each of the six groups which did not lose any $S$ through the application of this criterion, the one $S$ which was slowest on trials 5 through 8 was eliminated. In the seven groups which lost more than one $S$ through application of the criterion, enough replacements were run (a total of eight) to bring the $\mathrm{N}$ per group up to 15. This complicated procedure resulted in 20 groups of 15 each on which data were reported.

\section{Apparatus}

All training was in a 4-ft. enclosed runway, 4 in. wide, with a start box $8 \mathrm{in}$. long and $6 \mathrm{in}$. wide and a goal box $10 \mathrm{in.} \mathrm{long} \mathrm{and} 9$ in. wide. All parts of the apparatus were $8-1 / 2$ in. high and were painted black. The top of the alley was of hardware cloth, while the start box and goal box had Plexiglas lids. A guillotine door 4 in. from the end of the alley prevented retracing. A clock was started by the opening of the start-box door and stopped by the rat's weight on the goal-box floor.

\section{Procedure}

Each $S$ received seven days of prehandling and food scheduling followed by 32 days of acquisition. On each day of acquisition, all $20 \mathrm{Ss}$ in a squad were transported from the colony room to the experimental room in individual carrying cages with water bottles attached. Each $S$ received either four trials or four pseudotrials, with a minimum intertrial interval of $10 \mathrm{~min}$. On a pseudotrial, $\mathrm{S}$ was picked up, held for about $10 \mathrm{sec}$. and returned to the carrying cage. On a real trial, $S$ was placed in the start box and the door was opened as soon as $S$ oriented toward it. If $\mathrm{S}$ failed to enter the goal box within 2 min., $\mathrm{S}$ was placed in the goal box and a time of $120 \mathrm{sec}$. was recorded. The $S$ remained in the goal box for a minimum of $15 \mathrm{sec}$. and longer if necessary for all pellets to be eaten, up to a maximum of $3 \mathrm{~min}$., after which it was returned to the carrying cage. The pseudotrials were used to equate the amount of handling and general experimental experience for Ss differing in number of training trials. For the first 16 days, only the 128-trial groups received alley trials and all the other groups received pseudotrials. Each other group changed from pseudotrials to alley trials at the appropriate time so that all completed acquisition on the same day.

The Ss received their daily ration of $10 \mathrm{gm}$ of ground lab chow about $10 \mathrm{~min}$. after returning to their home cages in the colony room. For the 4-pellet and 8-pellet groups the ration was reduced enough to compensate 


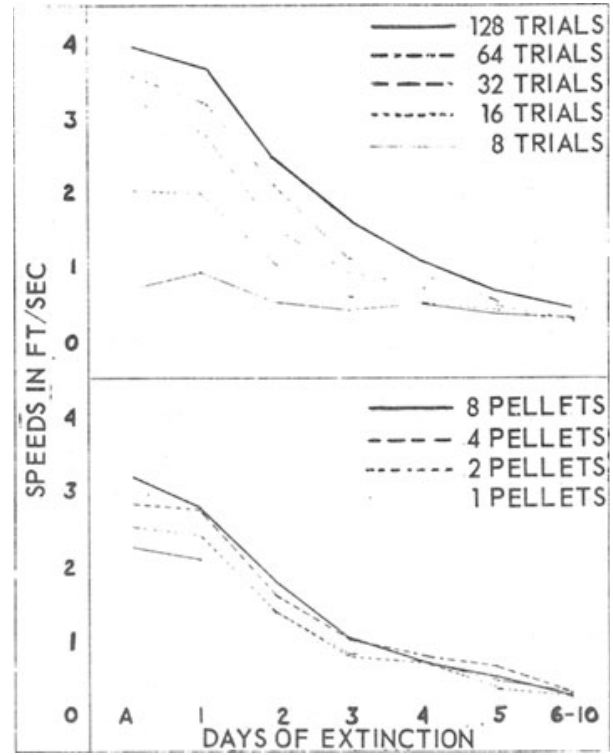

Fig. 1. Extinction curves for each level of acquisition training (upper graph, $\mathbf{N} \approx 60$ per curve) and each reward magnitude (lower graph, $N=75$ per curve). The last day of acquisition is designated by $A$, and the last five days of extinction are averaged.

approximately for the extra food they received in the alley.

On the day after the last day of acquisition, all groups began extinction, which lasted for 10 days at 4 trials a day. The procedure was the same as in acquisition except that neither food pellets nor food cup were present in the goal box.

\section{Resulis and Discussion}

A reciprocal transformation was used to convert all running times to speeds. Since most of the decline in speed took place during the first five days (20 trials) of extinction, separate analyses of variance were run on the last four trials of acquisition, the first 20 trials of extinction, and the last 20 trials of extinction.

Terminal acquisition speeds were a monotonic increasing function of number of trials $(F=113.36$, $\mathrm{df}=4 / 280, \mathrm{p}<.001)$ and of reward magnitude $(\mathrm{F}=13.92$, $\mathrm{df}=3 / 280, \mathrm{p}<.001)$, with no significant interaction $(F=1.31, \mathrm{df}=12 / 280)$. For the first 20 trials of extinction, speeds were again a monotonic increasing functuon of number of trials $(F=22.33, d f=4 / 280, p<.001)$. The effect of reward magnitude also tended to be an increasing function, but it was less regular than the effect of number of trials and fell short of statistical significance $(F=2.12, d f=4 / 280)$. The means showed a hint of the predicted interaction, in that the increasing effect of number of trials was slightly less for the largest than for the smallest reward magnitude, but there was no suggestion of statistical significance, the $F$ being less than one. For the last 20 trials of extinction, the effect of number of trials was mainly an increasing function, with a suggestion of a $U$ shape at the lower end $(F=2.57$, df $=4 / 280, p<.05)$. The $F$ values for reward magnitude and the interaction were both less than one. For these three analyses of variance, the mean squares within groups were, respectively, .94, .75, and .15. A supplementary analysis of Days 2-5 combined, using only the first and last trials of each day, revealed that the monotonic increasing relation between acquisition trials and extinction speeds applied to both the first and the last trials, but was significantly greater for the first trials (F for interaction $=12.47$, df $=4 / 280, p<.001$ ). There was no interaction between reward magnitude and trials.

Figure 1 shows speeds separately as a function of number of trials and of reward magnitude for the last day of acquisition, for each of the first five days of extinction, and for the last five days of extinction combined. It is evident that acquisition differences are maintained, with some minor reversals in the case of magnitude, until virtually all differences disappear by the end of 20 extinction trials. When these data are replotted as percentages of the speed on the last four trials of acquisition, except for the markedly greater resistance to extinction in the eight-trial gróups the differences among the various curves are so small as to render the various analyses suggested by Anderson (1963) superfluous.

Although the results of this experiment are fairly clearcut, when taken in conjunction with other studies they still leave in doubt the question of how reward magnitude and number of training trials affect extinction. Most puzzling is the contrast between the increasing effect of amount of training on extinction speed in this study and the decreasing effect under seemingly quite similar conditions in Ison's (1962) study. The interaction of reward magnitude and amount of training, if it has a consistent effect on extinction, evidently has it only under certain as yet undefined conditions, and the explanation of prior conflicting results remains to be determined.

\section{References}

Anderson, N. H. Comparison of different populations: Resistance to extinction and transfer. Psychol. Rev., 1963, 70, 162-179.

Hill, W. F., \& Spear, N. E. Resistance to extinction as a joint function of reward magnitude and the spacing of extinction trials. J. exp. Psychol., 1962, 64, 636-639.

Hulse, S. H., Jr. Amount and percentage of reinforcement and duration of goal confinement in conditioning and extinction. $J$. exp. Psychol., 1958, 56, 48-57.

Ison, J. R. Experimental extinction as a function of number of reinforcements. J. exp. Psychol., 1962, 64, 314-317.

Ison, J. R., \& Cook, P. E. Extinction behavior determined by number and magnitude of reinforcements. Paper read at $\mathrm{Mid}-$ western Psychological Association meeting, 1964.

Sperling, Sally E. Reversal learning and resistance to extinction: A review of the rat literature. Psychol. Bull., 1965, 63, 281-297.

\section{Notes}

1. This research was conducted at Northwestern University and and was supported by Public Hea!th Service Research Grant $\mathrm{MH}$ 10663 from the National Institute of Mental Health.

2. Currently at the Center for Advanced Study in the Behavioral Sciences. 